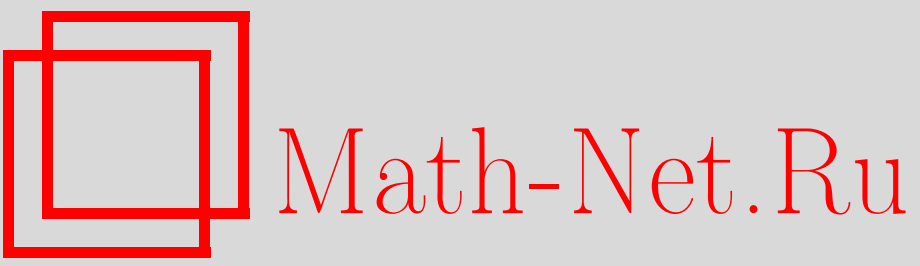

А. П. Янковский, Применение методов теории возмущений в плоской задаче равнонапряжённого армирования металлокомпозитных пластин при установившейся ползучести, Вестн. Сам. гос. техн. ун-та. Сер. Физ.-мат. науки, 2009, выпуск 2(), 53-71

DOI: https://doi.org/10.14498/vsgtu680

Использование Общероссийского математического портала Math-Net.Ru подразумевает, что вы прочитали и согласны с пользовательским соглашением

http://www. mathnet.ru/rus/agreement

Параметры загрузки:

IP : 3.80 .253 .173

26 апреля 2023 г., 16:59:39 
УДК 539.376

\title{
ПРИМЕНЕНИЕ МЕТОДОВ ТЕОРИИ ВОЗМУЩЕНИЙ В ПЛОСКОЙ ЗАДАЧЕ РАВНОНАПРЯЖЁННОГО АРМИРОВАНИЯ МЕТАЛЛОКОМПОЗИТНЫХ ПЛАСТИН ПРИ УСТАНОВИВШЕЙСЯ ПОЛЗУЧЕСТИ
}

\section{А. П. Янковский}

Институт теоретической и прикладной механики им. С. А. Христиановича СО РАН, 630090, г. Новосибирск, ул. Институтская, 4/1.

E-mails: nemirov@itam.nsc.ru, shulgin@itam.nsc.ru

\begin{abstract}
Методами теории возмущений решена задача равнонапряжённого армирования пластин, нагруженных в своей плоскости и работающих в условиях установившейся ползучести. Показана возможность существования нескольких альтернативных решений рассматриваемой задачи, которые можно надежно выделять, используя разработанный алгоритм. Построены конкретные проекты равнонапряжённого армирования двусвязной пластины при разных типах её нагружения.
\end{abstract}

Ключевые слова: пластины, установившаяся ползучесть, рациональное проектирование, равнонапряжённое армирование, малый параметр, теория возмущений.

Введение. При проектировании металлокомпозитных пластин целесообразно осуществлять их оптимизацию. Одним из прочностных критериев рационального армирования тонкостенных конструкций является требование равнонапряженности силовых элементов - волокон (проволок) - вдоль их траекторий, так как при этом несущая способность арматуры используется наиболее полно.

При длительной эксплуатации изделия в случае статического термосилового нагружения подавляющую часть времени металлокомпозитная конструкция работает в условиях установившейся ползучести [1], поэтому актуальной является проблема равнонапряженного армирования (РА) тонкостенных конструкций, работающих в условиях установившейся ползучести всех фаз композиции.

Постановка задачи РА металлокомпозитных пластин в условиях установившейся ползучести, нагруженных в своей плоскости и усиленных волокнами постоянного поперечного сечения, и математический анализ соответствующей системы разрешающих уравнений приведены в [2], где показано, что получающаяся система квазилинейных уравнений смешанно-составного типа и соответствующие ей нелинейные граничные условия не позволяют надеяться на построение широкого круга аналитических решений и требуют разработки обоснованных численных процедур решения. В то же время отмеченные в [2] особенности разрешающей системы уравнений не позволяют произвести простой перенос известных методов численного интегрирования квазилинейных систем уравнений [3] на решение рассматриваемой задачи. Наличие в задаче естественного малого параметра (см. ниже) позволяет надеяться на

Андрей Петрович Янковский (д.ф.-м.н.), ведущий научный сотрудник, лаборатория физики быстропротекающих процессов. 
эффективность применения хорошо зарекомендовавших себя методов теории возмущений [4 и др.].

Настоящая работа посвящается разработке и исследованию специфики применения методов теории возмущений для построения и анализа особенностей решений плоской задачи РА металлокомпозитных пластин в условиях установившейся ползучести.

1. Система разрешающих уравнений и граничные условия. Для удобства дальнейшего изложения систему разрешающих уравнений задачи РА и соответствующие ей граничные условия [2] будем записывать в безразмерном виде, предполагая, что в пластину постоянной толщины $h$ внедрено $N$ семейств армирующих проволок. В дальнейшем все размерные функции и величины будем помечать сверху чертой, а соответствующие им безразмерные функции и величины - обозначать теми же символами, но без черты. Тогда замкнутая система разрешающих уравнений, содержащая в качестве неизвестных функций безразмерные скорости $v_{1}, v_{2}$ перемещений при установившейся ползучести, безразмерную температуру $\theta$ и параметры PA $\omega_{k}, \psi_{k}$, состоит из [2]: уравнений равновесия

$$
(-1)^{i} \sum_{k} \sigma_{k} \omega_{k} l_{k j} \partial_{s}\left(\psi_{k}, \psi_{k}\right)+\varepsilon A_{i}(\boldsymbol{v}, \boldsymbol{\omega}, \theta)=-X_{i}(\boldsymbol{x}, \boldsymbol{\omega}), j=3-i, i=1,2 ;
$$

условий постоянства поперечных сечений волокон

$$
\partial_{s}\left(\psi_{k}, \omega_{k}\right)+\omega_{k} \partial_{n}\left(\psi_{k}, \psi_{k}\right)=0, \quad k \equiv 1,2, \ldots, N ;
$$

уравнения стационарной плоской теплопроводности

$$
\left(\Lambda_{11} \theta_{, 1}+\Lambda_{12} \theta_{, 2}\right)_{, 1}+\left(\Lambda_{21} \theta_{, 1}+\Lambda_{22} \theta_{, 2}\right)_{, 2}+\mu\left(\theta_{\infty}-\theta\right)=-Q(\boldsymbol{x}, \boldsymbol{\omega}) ;
$$

условий равнонапряженности арматуры

$$
\begin{gathered}
\partial_{s}\left(\psi_{k}, v_{1}\right) \cos \psi_{k}+\partial_{s}\left(\psi_{k}, v_{2}\right) \sin \psi_{k}=\xi_{k}(\theta)=f_{k}\left(\sigma_{k}, \theta\right) \\
\sigma_{k}=\mathrm{const}, \quad k \equiv 1,2, \ldots, N
\end{gathered}
$$

где

$$
\begin{gathered}
A_{i}(\boldsymbol{v}, \boldsymbol{\omega}, \theta)=2\left[a g_{0}(H, \theta)\left(2 v_{i, i}+v_{j, j}\right)\right]_{, i}+\left[a g_{0}(H, \theta)\left(v_{i, j}+v_{j, i}\right)\right],_{j}, \\
j=3-i, \quad X_{i}(\boldsymbol{x}, \boldsymbol{\omega}) \equiv X_{i}^{0}(\boldsymbol{x}) a+\sum_{k} X_{i}^{(k)}(\boldsymbol{x}) \omega_{k}, \quad i \equiv 1,2, \quad a=1-\Omega \\
\Omega=\sum_{k} \omega_{k}, \quad Q(\boldsymbol{x}, \boldsymbol{\omega}) \equiv a Q_{0}(\boldsymbol{x})+\sum_{k} \omega_{k} Q_{k}(\boldsymbol{x}), \quad l_{k 1}=\cos \psi_{k}, \quad l_{k 2}=\sin \psi_{k}, \\
\partial_{s}\left(\psi_{k}, \cdot\right)=(\cdot)_{, 1} \cos \psi_{k}+(\cdot)_{, 2} \sin \psi_{k}, \quad \partial_{n}\left(\psi_{k}, \cdot\right)=\partial_{s}\left(\psi_{k}+\pi / 2, \cdot\right)= \\
=-(\cdot)_{, 1} \sin \psi_{k}+(\cdot)_{, 2} \cos \psi_{k}, \quad k \equiv 1,2, \ldots, N, \quad \Lambda_{i j}=\Omega^{-1} \sum_{k} \omega_{k} \times \\
\times\left\{\left[\Omega\left(\lambda_{k}-\lambda_{0}\right)+\lambda_{0}\right] l_{k i} l_{k j}+(-1)^{i+j} l_{k s} l_{k r} \lambda_{k} \lambda_{0}\left[\Omega\left(\lambda_{0}-\lambda_{k}\right)+\lambda_{k}\right]^{-1}\right\} \\
s=3-i, \quad r=3-j, \quad i, j \equiv 1,2, \quad \boldsymbol{v}=\left\{v_{1}, v_{2}\right\}, \quad \boldsymbol{x}=\left\{x_{1}, x_{2}\right\} \\
\boldsymbol{\omega}=\left\{\omega_{1}, \omega_{2}, \ldots, \omega_{N}\right\}, \quad H=2 \sqrt{v_{1,1}^{2}+v_{1,1} v_{2,2}+v_{2,2}^{2}+0,25\left(v_{1,2}+v_{2,1}\right)^{2}}
\end{gathered}
$$


суммирование производится по указанному индексу от 1 до $N$; нижний индекс после запятой означает частное дифференцирование по соответствующей переменной $x_{i}$.

Пусть область $G$, занимаемая конструкцией в плане, ограничена контуром $\Gamma$, тогда на одной части (обозначим её $\Gamma_{p}$ ) этого контура могут быть заданы статические [2]

$$
\begin{aligned}
& \sum_{k} \sigma_{k} \omega_{k} \cos ^{2}\left(\psi_{k}-\beta\right)+\varepsilon D_{n}(\boldsymbol{v}, \boldsymbol{\omega}, \theta)=p_{n}, \\
& \sum_{k} \sigma_{k} \omega_{k} \sin 2\left(\psi_{k}-\beta\right)+\varepsilon D_{\tau}(\boldsymbol{v}, \boldsymbol{\omega}, \theta)=2 p_{\tau}, \quad\left(x_{1}, x_{2}\right) \in \Gamma_{p},
\end{aligned}
$$

где

$$
\begin{aligned}
& D_{n}(\boldsymbol{v}, \boldsymbol{\omega}, \theta)=2 a g_{0}(H, \theta)\left[v_{1,1}\left(1+n_{1}^{2}\right)+v_{2,2}\left(1+n_{2}^{2}\right)+\left(v_{1,2}+v_{2,1}\right) n_{1} n_{2}\right], \\
& D_{\tau}(\boldsymbol{v}, \boldsymbol{\omega}, \theta)=2 a g_{0}(H, \theta)\left[2\left(v_{2,2}-v_{1,1}\right) n_{1} n_{2}+\left(v_{1,2}+v_{2,1}\right)\left(n_{1}^{2}-n_{2}^{2}\right)\right], \\
& n_{1}=\cos \beta, n_{2}=\sin \beta,
\end{aligned}
$$

на другой части (обозначим её $\Gamma_{v}$ ) контура $\Gamma$ - кинематические

$$
v_{i}\left(\Gamma_{v}\right)=v_{i}^{0}\left(\Gamma_{v}\right), \quad i \equiv 1,2
$$

и на всем контуре $\Gamma\left(=\Gamma_{p} \cup \Gamma_{v}\right)$ - тепловые

$$
\chi\left[\left(\Lambda_{11} \theta_{, 1}+\Lambda_{12} \theta_{, 2}\right) n_{1}+\left(\Lambda_{21} \theta_{, 1}+\Lambda_{22} \theta_{, 2}\right) n_{2}+q\right]+\gamma\left(\theta-\theta_{0}\right)=0
$$

граничные условия. Кроме того, на части контура $\Gamma$ (обозначим её $\Gamma_{\omega k}$ ), на которой волокна $k$-того семейства «входят» в пластину, необходимо задать краевые условия для интенсивностей армирования [2]:

$$
\omega_{k}\left(\Gamma_{\omega k}\right)=\omega_{0 k}, \quad k \equiv 1,2, \ldots, N .
$$

Решение краевой задачи РА (1)-(4), (6), (8)-(10) должно удовлетворять физическим ограничениям [2]

$$
0 \leqslant \omega_{k}(k \equiv 1,2, \ldots, N), \quad 0 \leqslant \Omega \leqslant \omega_{*}<1 .
$$

В уравнениях и соотношениях (1)-(11) использованы следующие формулы обезразмеривания и обозначения:

$$
\begin{gathered}
\sigma_{k}=\bar{\sigma}_{k} / \bar{\sigma}_{1}^{*}, \quad \xi_{k}=\bar{\xi}_{k} / \bar{\xi}_{1}^{*}, \quad \lambda_{k}=\bar{\lambda}_{k} / \bar{\lambda}_{*}, \\
f_{k}\left(\sigma_{k}, \theta\right)=\bar{f}_{k}\left(\bar{\sigma}_{1}^{*} \sigma_{k}, \bar{\theta}_{*} \theta\right) / \bar{\xi}_{1}^{*}=\bar{f}_{k}\left(\bar{\sigma}_{k}, \bar{\theta}\right) / \bar{\xi}_{1}^{*}, \\
g_{0}(H, \theta)=\bar{g}_{0}\left(\bar{\xi}_{1}^{*} H, \bar{\theta}_{*} \theta\right) / \bar{B}_{*}=\bar{g}_{0}(\bar{H}, \bar{\theta}) / \bar{B}_{*}, \\
H=\bar{H} / \bar{\xi}_{1}^{*}, \quad v_{i}=\bar{v}_{i} /\left(\bar{D}_{1}^{*}\right), \quad x_{i}=\bar{x}_{i} / \bar{D} \\
X_{i}=\bar{D} \bar{X}_{i} / \bar{\sigma}_{1}^{*}, \quad X_{i}^{0}=\bar{D} \bar{X}_{i}^{0} / \bar{\sigma}_{1}^{*}, \quad X_{i}^{(k)}=\bar{D} \bar{X}_{i}^{(k)} / \bar{\sigma}_{1}^{*}, \\
\lambda_{0}=\bar{\lambda}_{0} / \bar{\lambda}_{*}, \quad \theta=\bar{\theta} / \bar{\theta}_{*}, \quad \theta_{0}=\bar{\theta}_{0} / \bar{\theta}_{*}, \quad \theta_{\infty}=\bar{\theta}_{\infty} / \bar{\theta}_{*}, \\
\left.Q=\bar{D}^{2} \bar{Q}_{(} / \bar{\lambda}_{*} \bar{\theta}_{*}\right), \quad Q_{k}=\bar{D}^{2} \bar{Q}_{k} /\left(\bar{\lambda}_{*} \bar{\theta}_{*}\right), \quad Q_{0}=\bar{D}^{2} \bar{Q}_{0} /\left(\bar{\lambda}_{*} \bar{\theta}_{*}\right), \\
q=\bar{D} \bar{q} /\left(\bar{\lambda}_{*} \bar{\theta}_{*}\right), \quad \mu=2 \bar{D}^{2} \bar{\mu} /\left(h \bar{\lambda}_{*}\right), \quad p_{n}=\bar{p}_{n} / \bar{\sigma}_{1}^{*}, \quad p_{\tau}=\bar{p}_{\tau} / \bar{\sigma}_{1}^{*}, \\
v_{i}^{0}=\bar{v}_{i}^{0} /\left(\bar{D} \bar{\xi}_{1}^{*}\right), \quad \Lambda_{i j}=\bar{\Lambda}_{i j} / \bar{\lambda}_{*}, \quad \varepsilon=\bar{B}_{*} / \bar{B}_{1}^{*}, \quad i, j \equiv 1,2, \quad k \equiv 1,2, \ldots, N ;
\end{gathered}
$$


$\bar{X}_{i}, \bar{v}_{i}$ - компоненты векторов приведённых объёмных (массовых) нагрузок и скорости установившейся ползучести точек пластины по направлениям $\bar{x}_{i}$ $(i \equiv 1,2)$ прямоугольной декартовой системы координат; $\bar{D}$-характерный размер пластины в плане; $\bar{X}_{i}^{0}, \bar{X}_{i}^{(k)}$ - компоненты удельных объёмных (массовых) нагрузок, действующих на связующее и арматуру $k$-того семейства; $\bar{\sigma}_{k}, \bar{\xi}_{k}$ - напряжение и скорость деформации ползучести в $k$-том семействе арматуры, связанные известной зависимостью $\bar{\xi}_{k}=\bar{f}_{k}\left(\bar{\sigma}_{k}, \bar{\theta}\right) ; \bar{g}_{0}(\bar{H}, \bar{\theta})$ - заданная функция, являющаяся коэффициентом пропорциональности между интенсивностью касательных напряжений $\bar{T}$ и интенсивностью скоростей деформаций $\bar{H}$ в связующем: $\bar{T}=\bar{g}_{0}(\bar{H}, \bar{\theta}) \bar{H} ; \omega_{k}, \psi_{k}$ - интенсивность и угол (отсчитываемый от направления $x_{1}$ ) армирования волокнами $k$-того семейства; $\omega_{*}$ - предельно допустимая удельная суммарная плотность армирования (на практике $\left.\omega_{*} \approx 0,7\right) ; \bar{\theta}, \bar{\theta}_{\infty}$ - температура конструкции и температура окружающей среды на лицевых плоскостях пластины; $\bar{\mu}$ - коэффициент конвективного теплообмена между связующим и окружающей средой на лицевых поверхностях пластины; $\bar{Q}_{0}, \bar{Q}_{k}$ - плотности мощностей внутренних источников тепла в связующем и волокнах $k$-того семейства; $\bar{Q}$ - приведённая плотность мощности внутренних источников тепла в композиции; $\bar{\lambda}_{0}, \bar{\lambda}_{k}$ - коэффициенты теплопроводности связующего и арматуры $k$-того семейства (в случае учёта термочувствительности фазовых материалов зависят от $\bar{\theta}) ; \bar{p}_{n}, \bar{p}_{\tau}$ нормальные и касательные контурные напряжения соответственно; $\bar{v}_{i}^{0}-$ заданные компоненты скорости перемещения точек контура $\Gamma_{v} ; \bar{\theta}_{0}-$ заданная на контуре $\Gamma$ температура; $\bar{q}$ - тепловой поток через торцевую (боковую) поверхность конструкции; $\chi, \gamma$ - функции переключения, позволяющие задавать на $\Gamma$ разные тепловые граничные условия; $\beta$ - угол, задающий направление внешней нормали к $\Gamma ; \omega_{0 k}$ - заданные на контуре $\Gamma_{\omega k}$ краевые значения функций $\omega_{k} ; \bar{\theta}_{*}$ - характерное значение температуры конструкции; $\bar{\lambda}_{*}-$ характерное значение коэффициента теплопроводности связующего; $\bar{\sigma}_{1}^{*}, \bar{\xi}_{1}^{*}-$ характерные значения напряжения и скорости деформации ползучести в первом семействе арматуры, связанные линейной зависимостью $\bar{\sigma}_{1}^{*}=\bar{B}_{1}^{*} \bar{\xi}_{1}^{*} ; \bar{B}_{*}$ характерное значение коэффициента пропорциональности $\bar{g}_{0}(\bar{H}, \bar{\theta})$ для связующего.

В случае степенного закона $\bar{\xi}=\bar{B} \bar{\sigma}^{m}$, связывающего скорость деформации $\bar{\xi}$ ползучести и напряжение $\bar{\sigma}$ в материале каждой фазы композиции, имеем зависимости [1]

$$
\begin{gathered}
\bar{g}_{0}(\bar{H}, \bar{\theta})=\bar{b}_{0}(\bar{\theta}) \bar{H}^{\mu_{0}(\bar{\theta})-1}, \\
\bar{b}_{0}=\left(\sqrt{3^{m_{0}+1}} \bar{B}_{0}\right)^{-\mu_{0}}, \quad \mu_{0}(\bar{\theta})=1 / m_{0}(\bar{\theta}), \\
\bar{f}_{k}\left(\bar{\sigma}_{k}, \bar{\theta}\right)=\bar{B}_{k}(\bar{\theta}) \bar{\sigma}_{k}^{m_{k}(\bar{\theta})}, \quad k \equiv 1,2, \ldots, N,
\end{gathered}
$$

где $\bar{B}_{0}(\bar{\theta}), m_{0}(\bar{\theta}), \bar{B}_{k}(\bar{\theta}), m_{k}(\bar{\theta})$ - известные характеристики установившейся ползучести материалов связующего и арматуры $k$-того семейства, зависящие заданным образом от температуры $\bar{\theta}$.

При выборе определенных материалов фаз композиции параметр $\varepsilon$ (см. (12)) может быть малым. Действительно, пусть металлокомпозитная пластина получена путём внедрения в медное $(\mathrm{Cu})$ связующее стальной проволоки 
У8А, тогда при температуре $\bar{\theta} \approx 200^{\circ} \mathrm{C}$ характеристики материалов фаз композиции (см. (13)) имеют значения $[5,6]$ :

$$
\begin{array}{rll}
\mathrm{Cu}: & m_{0}=2,16, & \bar{B}_{0}=5,63 \cdot 10^{-9}(\mathrm{M \Pi а})^{-m_{0}} \cdot \mathrm{ч}^{-1}, \\
\text { У8A: } & m_{1}=24,98, & \bar{B}_{1}=1,054 \cdot 10^{-84}(\mathrm{MПа})^{-m_{1}} \cdot \mathrm{ч}^{-1} .
\end{array}
$$

Аппроксимируем степенные законы установившейся ползучести рассматриваемых материалов с параметрами (14) двухзвенными кусочно-линейными функциями

$$
\bar{\sigma}_{k}=\left\{\begin{array}{cl}
\bar{B}_{k}^{(1)} \bar{\xi}_{k}, & 0 \leqslant \bar{\xi}_{k} \leqslant \bar{\xi}_{k}^{(1)}, \\
\bar{\sigma}_{k}^{(1)}+\bar{B}_{k}^{(2)}\left(\bar{\xi}_{k}-\bar{\xi}_{k}^{(1)}\right), & \bar{\xi}_{k}>\bar{\xi}_{k}^{(1)}>0, k \equiv 0,1 .
\end{array}\right.
$$

На рис. 1 кривые 1,2 характеризуют степенные зависимости $\bar{\sigma}_{k} \sim \bar{\xi}_{k}$ $(k \equiv 0,1)$ с параметрами (14) для меди и проволоки У8А соответственно. Двухзвенные ломаные $1^{\prime}, 2^{\prime}$ (штриховые линии) аппроксимируют кривые 1,2 и определяются параметрами (см. (15)):

$$
\begin{aligned}
\mathrm{Cu}: & \bar{B}_{0}^{(1)}=2,647 \cdot 10^{12} \Pi \mathrm{a} \cdot \mathrm{ч}, \quad \bar{\sigma}_{0}^{(1)}=49,005 \mathrm{M \Pi а}, \\
& \bar{\xi}_{0}^{(1)}=1,852 \cdot 10^{-5} \mathrm{ч}^{-1}, \quad \bar{B}_{0}^{(2)}=5,369 \cdot 10^{11} \Pi \mathrm{a} \cdot \text { ч; } \\
\text { У8A: } & \bar{B}_{1}^{(1)}=1,611 \cdot 10^{15} \Pi \mathrm{\Pi a} \cdot \bar{\sigma}_{1}^{(1)}=1,466 \text { ГПа, } \\
& \bar{\xi}_{1}^{(1)}=9,097 \cdot 10^{-7} \mathrm{ч}^{-1}, \quad \bar{B}_{1}^{(2)}=1,257 \cdot 10^{12} \Pi \mathrm{\Pi} \cdot \text { ч. }
\end{aligned}
$$

(Параметры (16) определены методом наименьших квадратов на интервале $0 \leqslant \bar{\xi} \leqslant 10^{-4}$ ч $^{-1}$, при этом варьировались величины $\bar{\sigma}_{k}^{(1)}, \bar{\xi}_{k}^{(1)}$. При принятом на рис. 1 масштабе по оси ординат линии $1,1^{\prime}$ визуально почти не отличаются.)

Если в (12) с учётом (16) принять $\bar{\sigma}_{1}^{*}=\bar{\sigma}_{1}^{(1)}, \bar{\xi}_{1}^{*}=\bar{\xi}_{1}^{(1)}, \bar{B}_{*}=\bar{B}_{0}^{(1)}, \bar{B}_{1}^{*}=$ $=\bar{B}_{1}^{(1)}$, то в рассматриваемой задаче $\varepsilon=\bar{B}_{*} / \bar{B}_{1}^{*}=0,00164-$ весьма малый параметр. (Подобные оценки малости параметра $\varepsilon$ можно получить и в случаях использования в качестве арматуры вольфрамовых проволок [7], а в качестве связующего - алюминиевых сплавов [8] и др.)

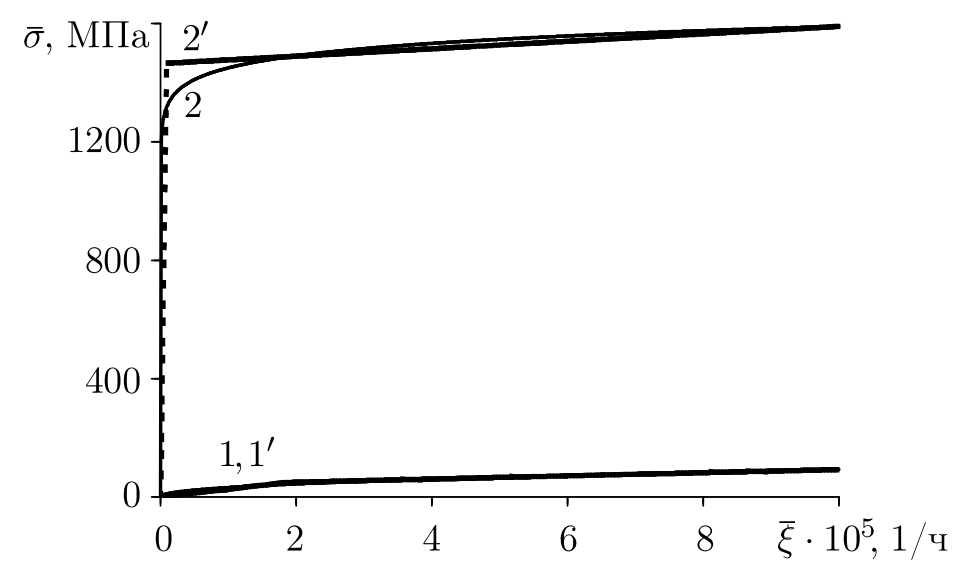

Рис. 1. Кривые установившейся ползучести фазовых материалов 
В [2] показано, что система разрешающих уравнений (1)-(4) является системой квазилинейных уравнений смешанно-составного типа [9], причём траектории армирования, определяемые углами $\psi_{k}$, являются её действительными характеристиками. Структура уравнений (1)-(4) и граничных условий (6) (с учётом соотношений $(5),(7))$ показывает, что задачи определения параметров РА $\psi_{k}, \omega_{k}$, температурного поля $\theta$ и поля скоростей $v_{1}, v_{2}$ установившейся ползучести связаны, и решать их необходимо совместно. Последнее обстоятельство существенно усложняет разработку методов решения нелинейной краевой задачи PA (1)-(4), (6), (8)-(10).

2. Применение методов теории возмущений. В настоящее время теория систем квазилинейных уравнений смешанно-составного типа разработана недостаточно полно [9], чтобы можно было аналитически исследовать свойства решений системы разрешающих уравнений (1)-(4) в общем случае, поэтому целесообразно воспользоваться какими-либо упрощениями этой системы и соответствующих ей граничных условий.

Важная особенность системы разрешающих уравнений задачи РА состоит в наличии в уравнениях равновесия (1) и в статических граничных условиях (6) малого параметра $\varepsilon$. Это позволяет разыскивать решение плоской задачи РА пластин при помощи методов теории возмущений [4 и др.], например, метода разложения решения в виде асимптотических рядов по степеням малого параметра $\varepsilon$ [4] или следующего итерационного процесса (который качественно схож с итерационным методом определения основного моментного напряженного состояния в теории оболочек [10]). Пусть $\psi_{k}^{(s)}, \omega_{k}^{(s)}, v_{i}^{(s)}$, $\theta^{(s)}(i \equiv 1,2,1 \leqslant k \leqslant N)-s$-тые приближения неизвестных функций, тогда $(s+1)$-е приближения будем получать путём интегрирования уравнений

$$
\begin{gathered}
(-1)^{i} \sum_{k} \sigma_{k} \omega_{k}^{(r)} l_{k j}^{(r)} \partial_{s}\left(\psi_{k}^{(r)}, \psi_{k}^{(r)}\right)=-X_{i}\left(\boldsymbol{x}, \boldsymbol{\omega}^{(r)}\right)-\varepsilon A_{i}\left(\boldsymbol{v}^{(s)}, \boldsymbol{\omega}^{(s)}, \theta^{(s)}\right) \\
j=3-i, \quad i \equiv 1,2 ; \\
\partial_{s}\left(\psi_{k}^{(r)}, \omega_{k}^{(r)}\right)+\omega_{k}^{(r)} \partial_{n}\left(\psi_{k}^{(r)}, \psi_{k}^{(r)}\right)=0, \quad k \equiv 1,2, \ldots, N \\
\left(\Lambda_{11}^{(r)} \theta_{, 1}^{(r)}+\Lambda_{12}^{(r)} \theta_{, 2}^{(r)}\right)_{, 1}+\left(\Lambda_{21}^{(r)} \theta_{, 1}^{(r)}+\Lambda_{22}^{(r)} \theta_{, 2}^{(r)}\right)_{, 2}+ \\
+\mu\left(\theta_{\infty}-\theta^{(r)}\right)=-Q\left(\boldsymbol{x}, \boldsymbol{\omega}^{(r)}\right) \\
\partial_{s}\left(\psi_{k}^{(r)}, v_{1}^{(r)}\right) \cos \psi_{k}^{(r)}+\partial_{s}\left(\psi_{k}^{(r)}, v_{2}^{(r)}\right) \sin \psi_{k}^{(r)}=\xi_{k}\left(\theta^{(r)}\right)=f_{k}\left(\sigma_{k}, \theta^{(r)}\right) \\
\sigma_{k}=\mathrm{const}, \quad k \equiv 1,2, \ldots, N, \quad\left(x_{1}, x_{2}\right) \in G
\end{gathered}
$$

при граничных и краевых условиях

$$
\begin{aligned}
\sum_{k} \sigma_{k} \omega_{k}^{(r)} \cos ^{2}\left(\psi_{k}^{(r)}-\beta\right) & =p_{n}-\varepsilon D_{n}\left(\boldsymbol{v}^{(s)}, \boldsymbol{\omega}^{(s)}, \theta^{(s)}\right) \\
\sum_{k} \sigma_{k} \omega_{k}^{(r)} \sin 2\left(\psi_{k}^{(r)}-\beta\right) & =2 p_{\tau}-\varepsilon D_{\tau}\left(\boldsymbol{v}^{(s)}, \boldsymbol{\omega}^{(s)}, \theta^{(s)}\right), \quad\left(x_{1}, x_{2}\right) \in \Gamma_{p} \\
v_{i}^{(m)}\left(\Gamma_{v}\right) & =v_{i}^{0}\left(\Gamma_{v}\right), \quad i \equiv 1,2
\end{aligned}
$$




$$
\begin{gathered}
\chi\left[\left(\Lambda_{11}^{(r)} \theta_{, 1}^{(r)}+\Lambda_{12}^{(r)} \theta_{, 2}^{(r)}\right) n_{1}+\left(\Lambda_{21}^{(r)} \theta_{, 1}^{(r)}+\Lambda_{22}^{(r)} \theta_{, 2}^{(r)}\right)\right. \\
\left.n_{2}+q\right]+ \\
+\gamma\left(\theta^{(r)}-\theta_{0}\right)=0 \\
\omega_{k}^{(r)}\left(\Gamma_{\omega k}\right)=\omega_{0 k}, \quad k \equiv 1,2, \ldots, N, \quad r=s+1 .
\end{gathered}
$$

Для начала итерационного процесса нулевое приближение выберем в виде

$$
v_{i}^{(0)}=0(i \equiv 1,2), \quad \theta^{(0)}=\mathrm{const} \approx 1 \quad\left(\text { например, } \theta^{(0)}=\bar{\theta}_{*} / \bar{\theta}_{*}=1\right) .
$$

Здесь $\boldsymbol{v}^{(s)}, \boldsymbol{\omega}^{(s)}$ - векторные функции, подобные $\boldsymbol{v}, \boldsymbol{\omega}\left(\right.$ см. (5)); $l_{k 1}^{(r)}=$ $=\cos \psi_{k}^{(r)}, l_{k 2}^{(r)}=\sin \psi_{k}^{(r)}$; выражения для коэффициентов $\Lambda_{i j}^{(r)}$ получаются из $\Lambda_{i j}$ (см. (5)) заменой $\psi_{k}, \omega_{k}$ их $r$-тыми приближениями $\psi_{k}^{(r)}, \omega_{k}^{(r)}$.

Уравнения итерационного процесса (17)-(23) получаются из соответствующих уравнений разрешающей системы следующим образом. В уравнениях равновесия (1) и в статических граничных условиях (6) в операторы $A_{i}$, $D_{n}, D_{\tau}$, имеющие в качестве сомножителя малый параметр $\varepsilon$, подставляются приближения неизвестных функций, определенные на предыдущей итерации. Поэтому слагаемые, имеющие сомножитель $\varepsilon$, на каждой итерации известны и в (17), (20) перенесены в правую часть. Задание начального приближения в виде (24) аналогично отбрасыванию в $(17),(20)$ на первой итерации $(r=1)$ слагаемых, содержащих $\varepsilon$. Остальные уравнения итерационного процесса на каждой итерации формально совпадают с соответствующими соотношениями разрешающей системы уравнений (см. п. 1).

Проанализируем систему (17)-(19) при различных количествах семейств арматуры $(N)$. Пусть в конструкцию внедрено одно семейство волокон $(N=1)$. Тогда три квазилинейных уравнения (17) содержат две неизвестные функции $\psi_{1}^{(r)}, \omega_{1}^{(r)}$, следовательно, подсистема (17) переопределена и для существования её решения необходимо выполнение некоторых условий совместности, которые свяжут между собой внешние нагрузки $X_{1}, X_{2}[11]$. С другой стороны, если система (17) совместна и её решение известно, то уравнение (18) замкнуто относительно приближения температуры $\theta^{(r)}$, но при $\psi_{1}^{(r)}, \theta^{(r)}$, известных из (17), (18), получаем одно линейное уравнение (19) относительно двух неизвестных функций $v_{1}^{(r)}, v_{2}^{(r)}$. Таким образом, подсистема (19) при $N=1$ оказывается незамкнутой относительно приближений скоростей установившейся ползучести, а значит, при использовании одного семейства волокон решение задачи РА методом (17)-(24) получить нельзя.

Если в конструкцию внедрены два семейства арматуры $(N=2)$, то для каждого $r \geqslant 1$ система (17)-(19) распадается на три подсистемы: первая подсистема (17) состоит из четырех квазилинейных уравнений и замкнута относительно приближений параметров РА $\psi_{k}^{(r)}, \omega_{k}^{(r)}$; вторая подсистема (18) состоит из одного уравнения и при $\psi_{k}^{(r)}, \omega_{k}^{(r)}$, известных из $(17)$, замкнута относительно приближения температуры $\theta^{(r)}$; третья подсистема (19) состоит из двух уравнений и при $\psi_{k}^{(r)}, \theta^{(r)}$, известных из $(17),(18)$, замкнута относительно приближений скоростей $v_{k}^{(r)}(k \equiv 1,2)$. Аналогично распадаются граничные и краевые условия $(20)-(23)$ : условия $(20),(23)$ состоят из четырёх 
равенств и замкнуты относительно контурных значений функций $\psi_{k}^{(r)}, \omega_{k}^{(r)}$; тепловое условие $(22)$ при $\psi_{k}^{(r)}, \omega_{k}^{(r)}$, известных из решения краевой задачи $(17),(20),(23)$, задаёт на Г граничные значения функции $\theta^{(r)}$ или условия по тепловому потоку, или граничные условия III рода, а кинематические условия (21) определяют на $\Gamma_{v}$ контурные значения двух функций $v_{k}^{(r)}(k \equiv 1,2)$.

Таким образом, при $N=2$ итерационный процесс (17)-(24) позволяет расщепить ранее связанные задачи определения параметров РА, температурного поля и поля скоростей установившейся ползучести на отдельные подзадачи, которые можно последовательно интегрировать на каждой итерации.

При внедрении в пластину более двух семейств арматуры $(N \geqslant 3)$ система (17)-(19) в целом замкнута относительно неизвестных функций, но её подсистема (17), содержащая только приближения параметров РА $\psi_{k}^{(r)}, \omega_{k}^{(r)}$ $(k \equiv 1,2, \ldots, N)$, не замкнута, так как состоит из $N+2$ уравнений и содержит $2 N$ неизвестных функций. Следовательно, при $N \geqslant 3$ система (17)-(19) не распадается так же просто, как это было при $N=2$, на отдельные подсистемы, определяющие приближения параметров РА, температуры и скоростей ползучести. При исследовании типа системы (17)-(19) выясняется, что её характеристический определитель тождественно равен нулю. Это вызвано тем, что $N(\geqslant 3)$ уравнений (19) содержат частные производные только от двух функций $v_{1}^{(r)}, v_{2}^{(r)}$, а значит, должны выполняться некоторые условия совместности этих уравнений. Покажем, что следствиями подсистемы (19) являются некоторые конечные и дифференциальные зависимости между приближениями углов РА $\psi_{k}^{(r)}(1 \leqslant k \leqslant N)$, которые определяют условия совместности этой системы. Для этого перепишем условия РА (19) в форме (см. (1.4) в [2])

$$
\xi_{11}^{(r)} l_{k 1}^{(r)} l_{k 1}^{(r)}+\xi_{22}^{(r)} l_{k 2}^{(r)} l_{k 2}^{(r)}+2 \xi_{12}^{(r)} l_{k 1}^{(r)} l_{k 2}^{(r)}=\xi_{k}^{(r)}, \quad k \equiv 1,2, \ldots, N,
$$

где

$$
\xi_{i j}^{(r)}=\frac{1}{2}\left(v_{i, j}^{(r)}+v_{j, i}^{(r)}\right), \quad \xi_{k}^{(r)} \equiv \xi_{k}\left(\theta^{(r)}\right) .
$$

Рассмотрим уравнения (25) как систему линейных алгебраических уравнений (СЛАУ) относительно $\xi_{i j}^{(r)}$. Выберем из (25) три уравнения, определитель которых отличен от нуля в некоторой окрестности точки $\left(x_{1}, x_{2}\right)$. Пусть для простоты это будут три первых уравнения. Тогда, используя правило Крамера, из них можно однозначно определить функции

$$
\xi_{i j}^{(r)}=\frac{\Delta_{i j}\left(\psi_{1}^{(r)}, \psi_{2}^{(r)}, \psi_{3}^{(r)}, \theta^{(r)}\right)}{\Delta\left(\psi_{1}^{(r)}, \psi_{2}^{(r)}, \psi_{3}^{(r)}\right)}, \quad i, j \equiv 1,2
$$

где

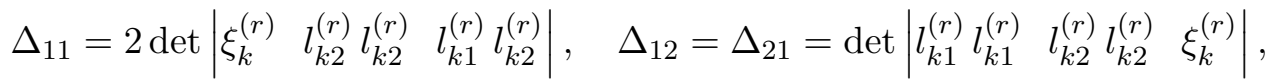

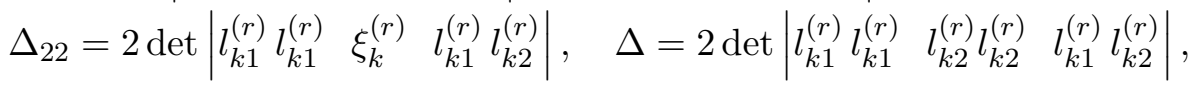

$$
\begin{aligned}
& k \equiv 1,2,3
\end{aligned}
$$


(здесь использована построчная запись определителей по $k$ ). Следовательно, приближения скоростей $\xi_{i j}^{(r)}$ деформаций ползучести являются нелинейными функциями от $\psi_{k}^{(r)}, \theta^{(r)}(k \equiv 1,2,3)$.

Если количество семейств арматуры больше трех, то, подставив выражения (27) в уравнения (25) при $4 \leqslant k \leqslant N$, получим $N-3$ конечных нелинейных зависимости между приближениями углов РА $\psi_{n}^{(r)}(1 \leqslant n \leqslant N)$ и температуры $\theta^{(r)}$. Кроме того, функции $\xi_{i j}^{(r)}$ должны удовлетворять безразмерному условию совместности скоростей деформаций ползучести [1]

$$
\xi_{11,22}^{(r)}+\xi_{22,11}^{(r)}=2 \xi_{12,12}^{(r)} .
$$

Подстановка выражений (27) в уравнение совместности (29) даёт ещё одну, но уже дифференциальную, зависимость между $\psi_{k}^{(r)}, \theta^{(r)}(k \equiv 1,2,3)$. Получающиеся при этом конечные и дифференциальные связи совместно с $(17),(18)$ образуют замкнутую систему уравнений относительно приближений параметров РА $\psi_{k}^{(r)}, \omega_{k}^{(r)}(k \equiv 1,2, \ldots, N)$ и температуры $\theta^{(r)}$, а для определения приближений скоростей $v_{1}^{(r)}, v_{2}^{(r)}$ ползучести можно использовать два любых уравнения (19) или первые соотношения (26) (в зависимости от вида кинематических граничных условий).

Следовательно, преобразованная таким образом система (17)-(19) позволяет вновь на каждой итерации расщепить ранее связанные подзадачи определения параметров РА, температурного поля и поля скоростей установившейся ползучести в пластине и интегрировать их последовательно. Однако основная трудность, возникающая при таком подходе, заключается в том, что при подстановке выражений (27) в уравнение (29) и при дальнейшем раскрытии определителей (28) получим квазилинейное уравнение, содержащее несколько тысяч слагаемых. Очевидно, что анализ такого уравнения потребует неоправданно больших затрат.

Таким образом, проведенное рассуждение показывает, что при внедрении в конструкцию более двух семейств арматуры итерационный процесс (17)(24) не приводит к принципиальному упрощению исходной системы разрешающих уравнений задачи РА.

Обратимся к более подробному анализу случая укладки двух семейств волокон, так как при этом, как было показано выше, итерационный процесс позволяет существенно упростить процедуру решения задачи РА. Далее в настоящем исследовании будем считать $N=2$. (С практической точки зрения условие $N=2$ не является обременительным, так как в композитных пластинах несущие элементы - арматура - должны воспринимать соизмеримые напряжения на площадках, различно ориентированных по двум направлениям.)

Правые части в двух первых уравнениях (17) можно трактовать как возмущенные объёмные (массовые) нагрузки, при этом система (17) формально совпадает с системой (3.4), (3.5) в [2], которая там подробно исследована. Здесь же отметим лишь основные свойства системы (17). Согласно [2], эта система квазилинейных уравнений с частными производными первого порядка имеет две двукратные действительные характеристики, определяемые 
углами $\psi_{k}^{(r)}(k \equiv 1,2)$, но не подпадает ни под один тип существующей на сегодняшний день классификации систем уравнений с частными производными [12]. Система (17) обладает свойствами, присущими как гиперболическим (ее нельзя считать гиперболической, так как она не приводится к характеристической форме $[2,13])$, так и параболическим (ее нельзя считать параболической, так как в общем случае в силу $\psi_{1}^{(r)} \neq \psi_{2}^{(r)}$ характеристики разных семейств различны) системам уравнений. Однако при отсутствии объёмных нагрузок $\left(X_{i} \equiv 0, i \equiv 1,2\right)$ на первой итерации $(r=1)$ в силу выбора начального приближения в виде (24) система (17) распадается на две замкнутые (при каждом $k$ ) подсистемы параболического типа (см. (3.14) в [2]). С другой стороны, характеристики системы (17) являются линиями слабых разрывов $[2,13]$, поэтому, например, решение задачи Коши для этой системы может быть получено лишь в области определенности решения (об этом свидетельствуют и многочисленные расчёты, проведенные автором), что характерно для решений задачи Коши для систем гиперболических уравнений [13].

Так как углы $\psi_{k}$ задают траектории РА, а $\psi_{k}^{(r)}-r$-тые приближения к $\psi_{k}$, то и характеристики системы (17) можно трактовать как $r$-тые приближения к траекториям армирования. Если итерационный процесс сходится, то характеристики системы (17) совпадут в пределе с траекториями РА, которые, в свою очередь, являются характеристиками системы разрешающих уравнений (1)-(4), как было показано в [2].

Для квазилинейных систем уравнений с действительными характеристиками наиболее естественно и просто ставится задача Коши, поэтому обратимся к вопросу постановки задачи Коши для системы (17). Эта система удовлетворяет условиям теоремы Коши-Ковалевской [12], а значит, при аналитических входных данных решение задачи Коши для нее существует и единственно. Для более широких классов входных данных теоремы существования для системы (17) автору не известны.

Покажем, что статические граничные условия (20) совместно с краевыми условиями (23) задают краевые условия для функций $\psi_{k}^{(r)}$. Для этого будем считать, что арматура обоих семейств «входит» в пластину на той части контура, где заданы статические граничные условия $\left(\Gamma_{p}=\Gamma_{\omega k}, k \equiv 1,2\right)$. Тогда систему трансцендентных уравнений $(20)$, замкнутую относительно $\psi_{k}^{(r)}\left(\Gamma_{p}\right)$ $(k \equiv 1,2)$, путём тригонометрических преобразований можно привести к виду

$$
\begin{aligned}
& \cos \left(2 \varphi_{2}-\psi\right)=\frac{b^{2}+c^{2}-\sigma_{1}^{2} \omega_{01}^{2}+\sigma_{2}^{2} \omega_{02}^{2}}{2 \sigma_{2} \omega_{02} \sqrt{b^{2}+c^{2}}}, \\
& \operatorname{tg} 2 \varphi_{1}=\frac{b-\sigma_{2} \omega_{02} \sin 2 \varphi_{2}}{c-\sigma_{2} \omega_{02} \cos 2 \varphi_{2}}, \\
& \psi_{k}^{(r)}\left(\Gamma_{p}\right)=\varphi_{k}+\beta \quad(k \equiv 1,2), \\
& \cos \psi=\frac{c}{\sqrt{b^{2}+c^{2}}}, \quad \sin \psi=\frac{b}{\sqrt{b^{2}+c^{2}}} \\
& b=2 p_{\tau}-\varepsilon D_{\tau}\left(\boldsymbol{v}^{(s)}, \boldsymbol{\omega}^{(s)}, \theta^{(s)}\right) \\
& c=2 P_{n}-\sigma_{1} \omega_{01}-\sigma_{2} \omega_{02}, \\
& P_{n}=p_{n}-\varepsilon D_{n}\left(\boldsymbol{v}^{(s)}, \boldsymbol{\omega}^{(s)}, \theta^{(s)}\right) \\
& r=s+1
\end{aligned}
$$


Функция $\beta$ в (30) задаёт направление внешней нормали к контуру $\Gamma_{p}$, поэтому, чтобы волокна входили на нем в область $G$, необходимо решение системы (30) разыскивать на открытом интервале $\varphi_{k} \in\left(\frac{\pi}{2}, \frac{3 \pi}{2}\right)$. На этом интервале первое уравнение (30) в зависимости от значений $\psi$ и правой части может иметь до двух различных корней, второе же уравнение при известном $\varphi_{2}$ имеет только один корень. Следовательно, система (30) может иметь до двух различных наборов решений относительно $\psi_{k}^{(r)}\left(\Gamma_{p}\right)$.

Таким образом, статические граничные условия и краевые условия для интенсивностей армирования задают два набора альтернативных краевых условий для системы (17), поэтому в классе аналитических входных данных задача Коши для этой системы может быть корректно поставлена на контуре $\Gamma_{p}=\Gamma_{\omega k}, k \equiv 1,2$.

При отсутствии распределённых объёмных нагрузок $\left(X_{i} \equiv 0, i \equiv 1,2\right)$ задача Коши $(17),(23),(30)$ на первой итерации $(r=1)$ может быть проинтегрирована аналитически (см. (3.14)-(3.24) в [2]). При этом из (17) с учётом (24) в общем случае $\sin \left(\psi_{1}^{(r)}-\psi_{2}^{(r)}\right) \neq 0$ следует

$$
\partial_{s}\left(\psi_{k}^{(r)}, \psi_{k}^{(r)}\right)=0, \quad k \equiv 1,2,
$$

а значит, характеристики системы (17) (а также и траектории РА в первом приближении) являются прямыми линиями, определяемыми контурными значениями углов $\psi_{k}^{(1)}\left(\Gamma_{p}\right)$, известными из $(30)$.

Замечание 1 . Как уже отмечалось, в общем случае нагружения пластины $\left(X_{i} \neq 0\right)$ теоремы существования решения задачи Коши $(17),(23),(30)$ в более широких, чем аналитический, классах входных данных автору не известны. Однако при отсутствии массовых нагрузок $\left(X_{i}=0, i \equiv 1,2\right)$ решение этой задачи Коши на первой итерации существует, если краевые значения для функций $\psi_{k}$ принадлежат классу гладких функций, а краевые значения для $\omega_{k}$ (т. е. $\left.\omega_{0 k}\right)$ принадлежат классу непрерывных функций, заданных на части контура $\Gamma_{p}$. (Об этом свидетельствует структура решения (3.19), (3.21)-(3.24) в [2].)

Если после интегрирования задачи Коши (17), (23), (30) её область определенности покрывает всю область $G$, занимаемую конструкцией в плане, то в каждой точке $\left(x_{1}, x_{2}\right) \in G$ известны функции $\psi_{k}^{(r)}, \omega_{k}^{(r)}$. При выполнении для функций $\omega_{k}^{(r)}$ ограничений, аналогичных (11), уравнение (18) является эллиптическим уравнением второго порядка [2] относительно приближения температуры $\theta^{(r)}$, которому соответствует тепловое граничное условие $(22)$. Такие граничные задачи и методы их интегрирования хорошо изучены $[3,14-$ 16], поэтому не будем останавливаться на обсуждении этих вопросов более подробно.

Если функции $\psi_{k}^{(r)}, \theta^{(r)}(k \equiv 1,2)$ известны из решения краевых задач $(17),(23),(30)$ и $(18),(22)$, то подсистема (19) является линейной гиперболической системой уравнений с частными производными первого порядка относительно приближений скоростей $v_{i}^{(r)}$ ползучести, причём её характеристики определяются углами $\psi_{k}^{(r)}$ и совпадают с характеристиками системы (17). В качестве краевых условий для подсистемы (19) выступают кинематические 
граничные условия (21), заданные на части контура $\Gamma_{v}$, где, по предположению, волокна «выходят» из пластины.

Так как область определенности решения задачи Коши (17), (23), (30) покрывает всю область $G$ и характеристики подсистем (17), (19) совпадают, то после интегрирования задачи Коши (19), (21) в каждой точке $\left(x_{1}, x_{2}\right) \in G$ будут известны функции $v_{i}^{(r)}, i \equiv 1,2$. (Методы интегрирования задачи Коши для гиперболических систем уравнений достаточно хорошо разработаны $[3,13]$, поэтому не будем останавливаться на этом вопросе более подробно.)

При отсутствии распределённых объёмных нагрузок $\left(X_{i}=0\right)$ и постоянстве температуры $\left(\theta^{(1)}=\theta_{0}=\right.$ const $)$ на первой итерации $(r=1)$ можно построить аналитическое решение задачи Коши (19), (21). Действительно, выше уже отмечалось, что при $X_{i}=0(i \equiv 1,2)$ характеристики системы (17), а значит, и системы (19) на первой итерации прямолинейны, т. е. выполняются равенства (31). Поэтому систему (19) при $\theta^{(1)}=\theta_{0}=$ const (в силу постоянства правых частей: $\xi_{k}=$ const) можно записать в форме инвариантов Римана [13] с нулевыми правыми частями, после чего легко построить решение задачи Коши (19), (21):

$$
\begin{aligned}
v_{1}^{(1)} \cos \psi_{k}^{(1)}+v_{2}^{(1)} & \sin \psi_{k}^{(1)}-\left(x_{1} \cos \psi_{k}^{(1)}+x_{2} \sin \psi_{k}^{(1)}\right) \xi_{k}= \\
& =v_{1}^{0}(\varsigma) \cos \psi_{k v}^{(1)}(\varsigma)+v_{2}^{0}(\varsigma) \sin \psi_{k v}^{(1)}(\varsigma)- \\
& -\left(\eta_{1}(\varsigma) \cos \psi_{k v}^{(1)}(\varsigma)+\eta_{2}(\varsigma) \sin \psi_{k v}^{(1)}(\varsigma)\right) \xi_{k}, \quad k \equiv 1,2,
\end{aligned}
$$

где $\xi_{k}=f_{k}\left(\sigma_{k}, \theta_{0}\right)=$ const, $\sigma_{k}=$ const; $\psi_{k v}^{(1)}(\varsigma)$ - значения функций $\psi_{k}^{(1)}$ на контуре $\Gamma_{v}$, известные из решения задачи Коши (17), (23), (30); $\eta_{k}(\varsigma)$ функции, задающие точки контура $\Gamma_{v}: x_{k}=\eta_{k}(\varsigma), k \equiv 1,2 ; \varsigma$ - естественный параметр вдоль контура. (Так как характеристики системы (19) при $X_{i}=$ $=0$ на первой итерации прямолинейны, то в уравнениях (32) имеют место равенства $\left.\psi_{k}^{(1)}\left(x_{1}, x_{2}\right)=\psi_{k v}^{(1)}(\varsigma).\right)$

Таким образом, на каждой итерации решение задачи РА сводится к последовательному интегрированию задачи Коши (17), (23), (30), граничной задачи (18), (22) и задачи Коши (19), (21). Такое неклассическое сведение краевой задачи РА к последовательности решений нескольких задач Коши возникает из-за того, что система разрешающих уравнений (1)-(4) совместно с граничными и краевыми условиями (6), (8)-(10) образуют краевую задачу с сингулярным возмущением [4], а итерационный процесс (17)-(24) относится к разряду методов теории возмущений.

Для проверки сходимости итерационного процесса целесообразно $r$-тые приближения неизвестных функций подставить в систему разрешающих уравнений и соответствующие ей граничные и краевые условия (п. 1), а затем проконтролировать нормы возникающих при этом невязок. Следует отметить, что невязки возникают лишь в уравнениях равновесия (1) и статических граничных условиях (6) и имеют вид:

$$
\begin{gathered}
\delta_{i}^{(r)}=\varepsilon\left[A_{i}\left(\boldsymbol{v}^{(r)}, \boldsymbol{\omega}^{(r)}, \theta^{(r)}\right)-A_{i}\left(\boldsymbol{v}^{(s)}, \boldsymbol{\omega}^{(s)}, \theta^{(s)}\right)\right], \\
\left(x_{1}, x_{2}\right) \in G, \quad i \equiv 1,2
\end{gathered}
$$




$$
\begin{gathered}
\delta_{n}^{(r)}=\varepsilon\left[D_{n}\left(\boldsymbol{v}^{(r)}, \boldsymbol{\omega}^{(r)}, \theta^{(r)}\right)-D_{n}\left(\boldsymbol{v}^{(s)}, \boldsymbol{\omega}^{(s)}, \theta^{(s)}\right)\right] ; \\
\delta_{\tau}^{(r)}=\varepsilon\left[D_{\tau}\left(\boldsymbol{v}^{(r)}, \boldsymbol{\omega}^{(r)}, \theta^{(r)}\right)-D_{\tau}\left(\boldsymbol{v}^{(s)}, \boldsymbol{\omega}^{(s)}, \theta^{(s)}\right)\right], \\
\left(x_{1}, x_{2}\right) \in \Gamma_{p} ; \quad r=s+1 .
\end{gathered}
$$

Эти равенства показывают, что определение невязок при использовании итерационного процесса не требует дополнительных вычислительных затрат, так как все выражения, входящие в (33), необходимо вычисляются для построения решения на $(r+1)$-й итерации. Автор считает, что на настоящий момент времени контроль норм невязок (33) является наиболее удобным практическим критерием проверки сходимости процесса (17)-(24).

ЗАмечАниЕ 2. Вместо кинематических граничных условий (8), (21) могут быть заданы некоторые смешанные условия, позволяющие определить скорости ползучести на контуре. Например, при задании на части $\Gamma_{s}$ контура $\Gamma$, кривизна $\kappa$ которой нигде не равна нулю, нормального напряжения $p_{n}$ и касательной скорости $v_{\tau}^{0}$ установившейся ползучести можно определить $r$-тое приближение для нормальной составляющей скорости $v_{n}$, если задача Коши $(17),(23),(30)$ уже проинтегрирована на $r$-той итерации. Действительно, используя на $\Gamma_{s}$ условия $\mathrm{PA}(25)(k \equiv 1,2)$ и первое статическое граничное условие (6), которое с учётом (7), (26) принимает вид

$$
\begin{aligned}
2 \varepsilon a^{(r)} g_{0}\left(H^{(r)}, \theta^{(r)}\right)[ & \left.\xi_{11}^{(r)}\left(1+n_{1}^{2}\right)+\xi_{22}^{(r)}\left(1+n_{2}^{2}\right)+2 \xi_{12}^{(r)} n_{1} n_{2}\right]= \\
& =p_{n}-\sum_{k} \sigma_{k} \omega_{k}^{(r)} \cos ^{2}\left(\psi_{k}^{(r)}-\beta\right), \quad\left(x_{1}, x_{2}\right) \in \Gamma_{s}
\end{aligned}
$$

можем, как из СЛАУ, определить $\xi_{i j}^{(r)}$ на части контура $\Gamma_{s}$. Тогда приближение скорости тангенциальной деформации ползучести на $\Gamma_{s}$ будет иметь вид

$$
\xi_{\tau \tau}^{(r)}=\xi_{11}^{(r)} \sin ^{2} \beta+\xi_{22}^{(r)} \cos ^{2} \beta-\xi_{12}^{(r)} \sin 2 \beta .
$$

Эта скорость деформации связана со скоростями точек контура $[1,17]$ :

$$
\xi_{\tau \tau}^{(r)}=\partial_{n}\left(\beta, v_{\tau}^{0}\right)+\kappa v_{n}^{(r)},
$$

где $v_{n}^{(r)}-r$-тое приближение нормальной составляющей вектора скорости смещений точек контура $\Gamma_{s}$. Если на $\Gamma_{s}$ всюду $\kappa \neq 0$, то из последнего равенства можно определить

$$
v_{n}^{(r)}=\frac{1}{\kappa}\left[\xi_{\tau \tau}^{(r)}-\partial_{n}\left(\beta, v_{\tau}^{0}\right)\right], \quad\left(x_{1}, x_{2}\right) \in \Gamma_{s}, \quad r=s+1 .
$$

Следовательно, задание на части контура $\Gamma_{s}$ нормального напряжения $p_{n}$ и касательной скорости $v_{\tau}^{0}$ ползучести позволяет на каждой итерации определить приближение нормальной составляющей $v_{n}^{(r)}$ скорости ползучести на 
этом контуре, а значит, и краевые условия для подсистемы (19), если кривизна части контура $\Gamma_{s}$, на которой волокна выходят из пластины, нигде не равна нулю. Подчеркнем, что в этом случае в окрестности контура $\Gamma_{s}$ может возникнуть краевой эффект (вследствие сингулярного возмущения исходной краевой задачи РА). Действительно, в уравнении (34) коэффициенты при $\xi_{i j}^{(r)}$ содержат в качестве сомножителя малый параметр $\varepsilon$, поэтому после разрешения СЛАУ $(25),(34)$ может оказаться, что $\xi_{i j}^{(r)}$ и $\xi_{\tau \tau}^{(r)}$ достигают по модулю больших значений на части контура $\Gamma_{s}$. Это обстоятельство может породить краевой эффект в связующем и отрицательно сказаться на сходимости итерационного процесса (17)-(24), (36). (Краевой эффект в арматуре не возникает, так как она всюду равнонапряжена: $\sigma_{k}=$ const, $k \equiv 1,2$.)

Так как равенства (23), (30) могут определять на контуре $\Gamma_{p}$ до двух альтернативных наборов краевых условий для системы (17) (выбор того или иного набора этих условий определяется только волей проектировщика или из каких-либо дополнительных соображений), удовлетворяющей условиям теоремы Коши-Ковалевской [12], а также в силу единственности решения граничной задачи (18), (22) [14] и задачи Коши (19), (21) [13] при помощи итерационного метода (17)-(24) можно построить до двух различных решений исходной задачи РА, если, конечно, они существуют. Этими решениями можно управлять за счет варьирования контурных функций $\omega_{0 k}$ в краевых условиях (23). (Так как выбор того или иного решения всецело зависит от воли проектировщика, то в процессе решения задачи РА при использовании разработанного итерационного метода не может произойти «несанкционированного» перехода от одного решения к другому, например за счет погрешности численных схем и т. п.) Такое многообразие альтернативных проектов РА выгодно с практической точки зрения, так как позволяет выбрать из их совокупности проекты, обладающие теми или иными эксплуатационными свойствами, а также наиболее удобные с точки зрения технологической реализации.

ЗАмЕчАние 3. Если в пластине с РА-структурой оба семейства волокон изготовлены из одного материала $\left(f_{1}(\sigma, \theta)=f_{2}(\sigma, \theta)\right)$ и напряжения в них одинаковы $\left(\sigma_{1}=\sigma_{2}\right)$, а на контуре $\Gamma_{p}=\Gamma_{\omega k}$ в краевых условиях (23) выполняются равенства $\omega_{01}=\omega_{02}$, то РА-структура в обоих возможных проектах совпадает. (С математической точки зрения два решения отличаться лишь тем, что траектории РА разных семейств меняются в них местами; с технологической точки зрения это один и тот же проект.)

ЗАмечАние 4. Как уже отмечалось в начале настоящего пункта, решение рассматриваемой задачи РА можно построить и с помощью разложения всех неизвестных функций в асимптотические ряды по малому параметру [4]

$$
\begin{gathered}
\psi_{k}(\boldsymbol{x})=\sum_{m=1}^{\infty} \psi_{k}^{(m)}(\boldsymbol{x}) \varepsilon^{m-1}, \quad \omega_{k}(\boldsymbol{x})=\sum_{m=1}^{\infty} \omega_{k}^{(m)}(\boldsymbol{x}) \varepsilon^{m-1}, \\
\theta(\boldsymbol{x})=\sum_{m=1}^{\infty} \theta^{(m)}(\boldsymbol{x}) \varepsilon^{m-1}, \quad v_{i}(\boldsymbol{x})=\sum_{m=1}^{\infty} v_{i}^{(m)}(\boldsymbol{x}) \varepsilon^{m-1}, \\
i \equiv 1,2, \quad k \equiv 1,2, \ldots, N .
\end{gathered}
$$

Асимптотический анализ системы разрешающих уравнений (1)-(4) и соответствующих ей граничных (6), (8), (9) и краевых (10) условий с учётом 
разложений (37) показывает:

1) первые слагаемые $\left(\psi_{k}^{(1)}, \omega_{k}^{(1)}, \theta^{(1)}, v_{i}^{(1)}\right)$ в рядах $(37)$ полностью совпадают с решением системы $(17)-(24)$ на первой $(r=1)$ итерации;

2 ) если на второй $(r=2)$ итерации метода $(17)-(24)$ левые части уравнений (17)-(20), (22) линеаризовать в окрестности решения для первой итерации, то получающаяся система и краевые условия будут совпадать с уравнениями и краевыми условиями, определяющими коэффициенты при вторых слагаемых $\left(\psi_{k}^{(2)}, \omega_{k}^{(2)}, \theta^{(2)}, v_{i}^{(2)}\right)$ в разложениях $(37)$. На основе столь существенного сходства уравнений итерационного процесса (17)-(24) и метода асимптотических рядов (37) все результаты исследований, полученные ранее в настоящем пункте при анализе уравнений (17)-(24), можно перенести и на соответствующие цепочки уравнений, получающиеся после подстановки (37) в исходную систему разрешающих уравнений и краевые условия задачи РА (п. 1). Однако итерационный процесс (17)-(24) обладает рядом преимуществ перед методом асимптотических рядов:

1) правые части в уравнениях (17)-(23) на каждой итерации имеют одну и ту же достаточно простую структуру (в цепочках же уравнений метода асимптотических рядов (37) структура правых частей с увеличением номера уточнения $m$ существенно усложняется);

2 ) для построения $r$-того приближения по методу итераций используется информация лишь о предыдущем $(r-1)$-м приближении (в правых же частях уравнений метода асимптотических рядов используется информация о всех предыдущих $n$-тых уточнениях, $n \equiv 1,2, \ldots, r-1)$;

3) характеристики подсистем (17), (19) изменяются от итерации к итерации и при сходящемся итерационном процессе совпадают в пределе с траекториями армирования (в уравнениях же метода асимптотических рядов (37) характеристики в соответствующих подсистемах уравнений фиксированы и совпадают с характеристиками подсистем (17), (19) на первой $(r=1)$ итерации);

4) для проверки сходимости обоих обсуждаемых методов целесообразно $r$-тое приближения по методу итераций и $r$-е частичные суммы типа (37) подставить в систему разрешающих уравнений и соответствующие ей краевые условия (п. 1) и проконтролировать нормы возникающих при этом невязок, причём в случае метода асимптотических рядов (37) эти невязки возникают во всех уравнениях и краевых условиях исходной системы (п. 1) и с увеличением номера уточнения (с увеличением $r$ ) структура их существенно усложняется, в случае же итерационного процесса невязки возникают только в уравнениях $(1),(6)$ и имеют достаточно простую структуру (33).

Указанные преимущества позволяют отдать предпочтение итерационному процессу (17)-(24) при практическом решении задач РА пластин в условиях установившейся ползучести.

ЗАмечАние 5. Если задача РА уже решена (известны функции $\psi_{k}, \omega_{k}, \theta$, $\left.v_{k}, k \equiv 1,2\right)$, то по формулам (1.2)-(1.4) из [2] можно определить напряженное состояние в связующем и в композиции $\left(\sigma_{i j}\right)$ пластины в условиях установившейся ползучести. Кроме того, зная $\psi_{k}, \omega_{k}, \theta(k \equiv 1,2)$, можно определить и начальное напряженное состояние $\bar{\sigma}_{i j}$ в пластине (его можно вычислить, например, методами, изложенными в $[18,19])$. Решение же о неустановившейся 
ползучести на первой её стадии можно получить приближённо, используя методику, предложенную в [1]. Для этого представим текущие осреднённые напряжения в пластине в виде

$$
\overline{\bar{\sigma}}_{i j}=(1-\tau(t)) \bar{\sigma}_{i j}+\tau(t) \sigma_{i j}, \quad i, j \equiv 1,2,
$$

где $\tau(t)$ - монотонная функция времени $t$, возрастающая от значения $\tau=0$ в начальный момент времени $t=0$ до $\tau=1$ при $t \rightarrow \infty$. Функцию $\tau(t)$ можно определить исходя из экстремальных принципов теории ползучести (см. § 34 в [1]). В случае изотропных пластин реализация такого подхода не вызывает особых трудностей (так как можно почти дословно повторить все рассуждения $\S 34$ в [1]), а в случае сложно армированных пластин (как это имеет место в задачах РА) требует проведения дополнительного более тщательного исследования, выходящего за рамки настоящей работы.

3. Обсуждение результатов расчётов. В качестве примера рассмотрим расчёт РА-структур при установившейся ползучести металлокомпозитной двусвязной пластины, ограниченной контурами, уравнения которых заданы в безразмерной полярной $(r, \phi)$ системе координат:

$$
\begin{aligned}
\Gamma_{p}=\Gamma_{\omega 1}=\Gamma_{\omega 2}: & r_{1}(\varphi)=0,5-0,05 \cos (2 \varphi-\pi / 2), \\
\Gamma_{v}: & r_{2}(\varphi)=1+0,08 \cos (2 \varphi), \quad 0 \leqslant \varphi<2 \pi .
\end{aligned}
$$

Внешний контур $\Gamma_{v}$ пластины жестко закреплен $\left(v_{1}^{0}(\varphi)=v_{2}^{0}(\varphi)=0\right)$, а на внутреннем контуре $\Gamma_{p}$ заданы равномерно распределённые контурные нагрузки $p_{n}$ или $p_{\tau}$. Распределенные объёмные нагрузки отсутствуют $\left(X_{i} \equiv 0\right.$, $i \equiv 1,2)$, температурное поле предполагается однородным $\left(\theta=\theta_{0}=\right.$ const $)$. Пластина изготовлена из меди и армирована двумя семействами стальной проволоки У8А (механические характеристики установившейся ползучести фаз композиции имеют значения $(13),(14))$. Плотности армирования проволками обоих семейств на внутреннем контуре имеют значения

$$
\omega_{1}\left(\Gamma_{p}\right)=\omega_{2}\left(\Gamma_{p}\right)=\omega_{01}(\varphi)=\omega_{02}(\varphi)=0,35, \quad \Gamma_{p}=\Gamma_{\omega 1}=\Gamma_{\omega 2} .
$$

На рис. 2 изображена РА-структура, полученная в случае нагружения внутреннего контура давлением $\bar{p}_{n}=-500 \mathrm{MПа}\left(\bar{p}_{\tau}=0\right)$ при задании уровня напряжений в арматуре $\bar{\sigma}_{1}=\bar{\sigma}_{2}=-1$ ГПа. Согласно замечанию 3 , в этом случае структура, соответствующая второму решению задачи РА, полностью совпадает с изображенной на рис. 2. (Об этом свидетельствуют и результаты проведенного расчёта.)

На рис. 3 изображены две альтернативные РА-структуры, полученные в случае нагружения внутреннего контура касательной нагрузкой $\bar{p}_{\tau}=$ $=-250 \mathrm{MПа}\left(\bar{p}_{n}=0\right)$ при задании уровня напряжений в арматуре $\bar{\sigma}_{1}=$ $=-\bar{\sigma}_{2}=1$ ГПа. При этом существуют оба решения задачи РА, и, как видно из рис. 3, структуры армирования в них существенно отличаются. Однако, кроме структуры, отличие заключается и в напряженном состоянии, возникающем в связующем. Так, в проекте, изображенном на рис. $3, \sigma$, максимум интенсивности напряжений в связующем на $43,75 \%$ больше той же величины в проекте, представленном на рис. 3, а. Кроме того, в указанных проектах отличаются поля скоростей установившейся ползучести и общий расход арматуры, который в случае, изображенном на рис. 3, б, на 44,28\% меньше, чем в РА-структуре, приведённой на рис. 3, а. 


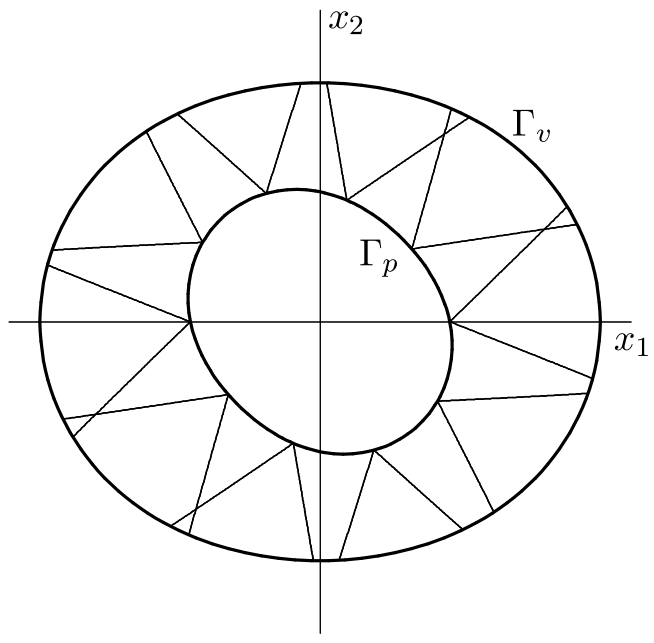

Рис. 2. Структура равнонапряженного армирования двусвязной пластины, нагруженной на внутреннем контуре давлением

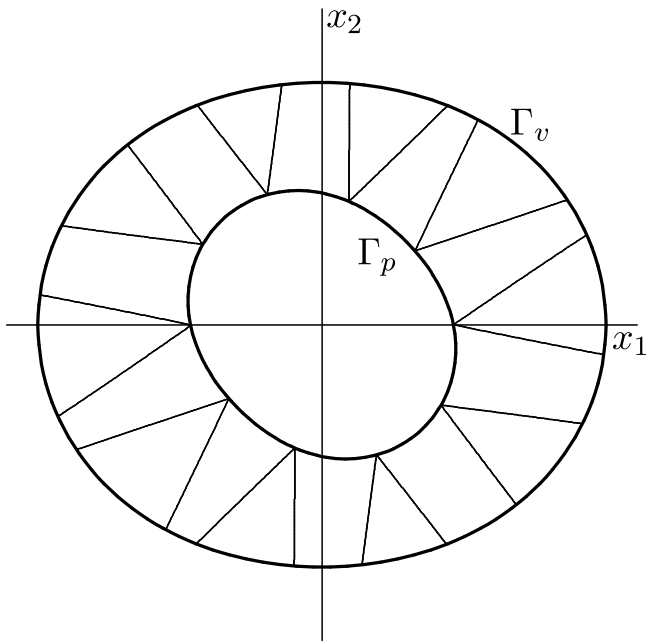

a

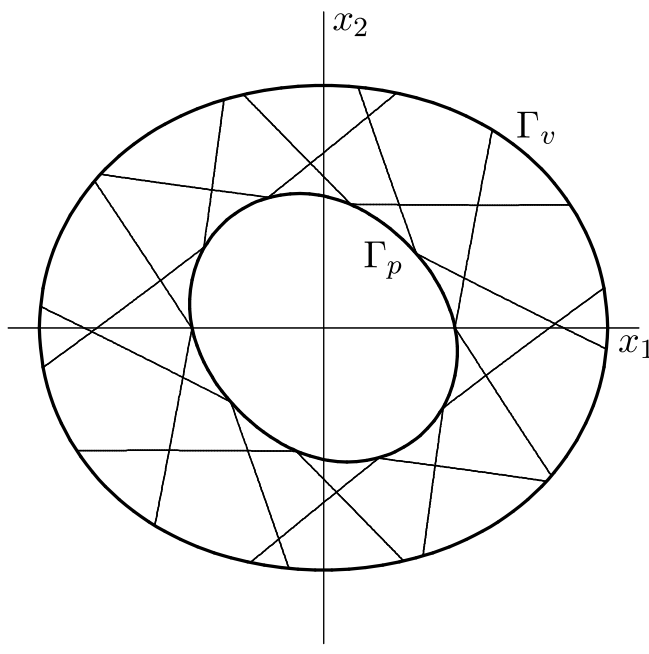

б

Рис. 3. Два альтернативных проекта равнонапряженного армирования двусвязной пластины, нагруженной на внутреннем контуре касательными нагрузками

Подчеркнём, что возможность построения двух альтернативных РА-проектов определяется тем, что задачи РА относятся к разряду обратных задач механики композитов (а точнее, к специфическим коэффициентным задачам), которые, как известно, могут иметь неединственное решение.

Возможность построения двух решений задачи РА можно продемонстрировать на следующем простейшем примере. Рассмотрим прямоугольную удлиненную пластину (в идеале бесконечной длины) шириной $l$, в которой закрепление, нагружение, температурное поле и структура не изменяются в продольном направлении $x_{2}$. (Пренебрегая торцевыми эффектами, в этом случае получим решение задачи РА, зависящее только от переменной $x_{1}$.) Тогда при однородном температурном поле $\left(\theta=\theta_{0}=\mathrm{const}\right)$, отсутствии объёмных 
нагрузок $\left(X_{i} \equiv 0, i \equiv 1,2\right)$ и задании на продольном контуре $x_{1}=l>0$ постоянной касательной нагрузки $p_{\tau}=$ const $\neq 0\left(p_{n}=0\right)$ из $(1),(6),(7)$ получим $(\beta=0)$

$$
\begin{aligned}
& \sum_{k} \sigma_{k} \omega_{k} \cos ^{2} \psi_{k}+4 \varepsilon a g_{0}(H, \theta) v_{1,1}=p_{n}=0, \\
& \sum_{k}^{k} \sigma_{k} \omega_{k} \sin 2 \psi_{k}+2 \varepsilon a g_{0}(H, \theta) v_{2,1}=2 p_{\tau}, \\
& \theta=\theta_{0}=\mathrm{const}, \quad 0 \leqslant x_{1} \leqslant l .
\end{aligned}
$$

Условие РА (4) принимает вид

$$
v_{1,1} \cos ^{2} \psi_{k}+0,5 v_{2,1} \sin 2 \psi_{k}=\xi_{k}=\text { const }, \quad k \equiv 1,2 .
$$

Рассмотрим PA-структуры, в которых $\omega_{1}=\omega_{2}, \psi_{1}=-\psi_{2}, \sigma_{1}=-\sigma_{2}, \xi_{1}=$ $=-\xi_{2}$, тогда из (39) получим $v_{1,1}=0$ и первое уравнение (38) выполняется тождественно, а второе преобразуется в

$$
\sigma_{1} \omega_{1} \sin 2 \psi_{1}+\varepsilon a g_{0}\left(H, \theta_{0}\right) v_{2,1}=p_{\tau}, \quad 0 \leqslant x_{1} \leqslant l .
$$

В силу малости параметра $\varepsilon$ отбросим в первом приближении второе слагаемое в левой части равенства (40), тогда получим

$$
\sigma_{1} \omega_{1} \sin 2 \psi_{1}=p_{\tau}, \quad 0 \leqslant x_{1} \leqslant l .
$$

Отсюда, задав изначально $p_{\tau}, \sigma_{1}$ и $\omega_{1}$ (так как $\omega_{1}=\omega_{01}=$ const, cм. (10)), можем определить угол армирования

$$
\psi_{1}=\frac{1}{2} \arcsin \left(\frac{p_{\tau}}{\sigma_{1} \omega_{1}}\right), \quad 0 \leqslant x_{1} \leqslant l
$$

Однако помимо (42) решением уравнения (41) является и $\bar{\psi}_{1}=\frac{\pi}{2}-\psi_{1}$, причём эти решения совпадают лишь в частном случае $\bar{\psi}_{1}=\psi_{1}=\frac{\pi}{4}$, т. е., согласно (42), при $p_{\tau}=\sigma_{1} \omega_{1}$. В общем же случае $\left(\left|p_{\tau}\right|<\left|\sigma_{1} \omega_{1}\right|\right)$ уравнение (41) имеет два альтернативных решения $\left(\bar{\psi}_{1} \neq \psi_{1}\right)$, которым соответствуют две различных РА-структуры (как это имеет место на рис. 3).

Работа выполнена при поддержке РФФИ (код проекта 07-08-00152-а).

\section{БИБЛИОГРАФИЧЕСКИЙ СПИСОК}

1. Качанов Л. М. Теория ползучести. - М.: Физматгиз, 1960. - 456 с.

2. Немировский Ю. В., Янковский А. П. Равнонапряженное армирование металлокомпозитных пластин волокнами постоянного поперечного сечения в условиях установившейся ползучести // Механика композитных материалов, 2008. - Т. 44, № 1. - С. 1134 .

3. Самарский A. А. Теория разностных схем. - М.: Наука, 1989. - 616 с

4. Найбе А. Введение в методы возмущений. - М.: Мир, 1984. - 536 с.

5. Писаренко Г. С., Можаровский Н. С. Уравнения и краевые задачи теории пластичности и ползучести: Справочное пособие. - Киев: Наукова думка, 1981. - 496 с.

6. Композиционные материалы: Справочник / ред. Д. М. Карпинос. - Киев: Наукова думка, 1985. - $592 \mathrm{c.}$

7. Карпинос Д. М., Невгод В.А., Тучинский Л. И. и др. Ползучесть и длительная прочность вольфрамовых проволок // Пробл. прочности, 1972. - № 1. - С. 70-73.

8. Соснин О.В. О ползучести слабо упрочняющихся материалов при нестационарных температурно-силовых режимах // Пробл. прочности, 1972. - №1. - С. 74-77. 
9. Джураев Т. Д. Краевые задачи для уравнений смешанного и смешанно-составного типов. - Ташкент: Фан, 1979. - 238 с.

10. Новожилов В. В., Черных К. Ф., Михайловский Е. И. Линейная теория тонких оболочек. - Л.: Политехника, 1991. - 656 с.

11. Камке Э. Справочник по дифференциальным уравнениям в частных производных первого порядка. - М.: Наука, 1966. - 260 с.

12. Петровский И. Г. Лекции об уравнениях с частными производными. - М.: Физматгиз, 1961. - $400 \mathrm{c}$.

13. Рождественский Б. Л., Яненко Н. Н. Системы квазилинейных уравнений. - М.: Наука, 1969. - 592 с.

14. Бицадзе А. В. Краевые задачи для эллиптических уравнений второго порядка. - М.: Наука, 1966. - 204 с.

15. Беляев H. М., Рядно А. А. Методы теории теплопроводности: Учеб. пособие для вузов. В 2-х частях. Ч. 1. - М.: Высш. школа, 1982. - 327 с.

16. Беляев H. М., Рядно А. А. Методы теории теплопроводности: Учеб. пособие для вузов. В 2-х частях. Ч. 2. - М.: Высш. школа, 1982. - 304 с.

17. Демидов С. П. Теория упругости. - М.: Высш. школа, 1979. - 432 с.

18. Немировский Ю. В., Резников Б. С. Прочность элементов конструкций из композитных материалов. - Новосибирск: Наука, 1986. - 168 с.

19. Немировский Ю. В., Янковский А. П. Термоупругопластический изгиб сложно армированных пластин // Механика композитных материалов, 2005. - Т. 41, №6. - С. 715742 .

MSC: 74K20, 74R20

\section{APPLICATION OF METHODS OF THE THEORY OF PERTURBATIONS IN FLAT PROBLEM OF EQUALLY-STRESSED REINFORCING OF METAL-COMPOSITE PLATES AT STEADY CREEP}

\section{A.P. Yankovskii}

Khristianovich Institute of Theoretical and Applied Mechanics,

Siberian Branch of the Russian Academy of Sciences,

630090, Novosibirsk, Institutskaya st., 4/1.

E-mails: nemirov@itam.nsc.ru, shulgin@itam.nsc.ru

The problem of equally-stressed reinforcings of the plates loaded in their plane and working in condition of steady creep is solved by methods of the theory of perturbations. The possibility of existence of several alternative solutions of the problem under consideration is demonstrated which can be selected reliably, using the algorithm developed. Explicit projects of equally-stressed reinforcings of a doubly-connected plate are constructed at different types of its load.

Key words: plates, steady creep, rational projection, equal-stress reinforcing, small parameter, perturbations theory.

Original article submitted 27/II/2009; revision submitted 16/VIII/2009.

Andrey P. Yankovskii (Dr. Sci. (Phys. \& Math.)), Leading Research Scientist, Physics of Fast Process Laboratory. 